\title{
Bio-electrosprayed human sperm remain viable
}

Helen C. O’Neill' ${ }^{1}$, Walid Maalouf ${ }^{2}$, Joyce Harper ${ }^{1}$, Suwan N. Jayasinghe ${ }^{3 *}$

${ }^{1}$ Dr. Helen C. O’Neill and Professor Joyce Harper

Embryology, IVF and Reproductive Genetics Group, Institute for Women's Health

University College London

86-96 Chenies Mews

London WC1E 6HX

United Kingdom

${ }^{2}$ Dr. Walid Maalouf

Developmental Programming Group

Division of Child Care Health

Obstetrics and Gynecology

Queen's Medical Centre

Nottingham NG7 2UH

United Kingdom

${ }^{3}$ Professor Suwan N. Jayasinghe*

BioPhysics Group,

Institute of Biomedical Engineering, Centre for Stem Cells and Regenerative Medicine and Department of Mechanical Engineering

University College London

Torrington Place

London WC1E 7JE

United Kingdom

E-mail: s.jayasinghe@ucl.ac.uk 
In our previous investigations, we demonstrated that certain living cells exposed to bioelectrosprays remained viable, and behaved as expected in comparison to control cells. These studies also extended to post-bio-electrosprayed cells being transplanted into mice, which demonstrated no rejection, and in fact they were seen to integrate with the surrounding host tissues. Therefore, highlighting bio-electrosprays as a front running bioplatform for engineering functional tissues for repair, replacement and rejuvenation of damaged and/ageing tissues. In the present studies, we take bio-electrosprays further into human health, investigating the possibility of this platform biotechnology to directly handle the smallest and most highly specialised cell in the human body, the spermatozoon. These studies demonstrated the ability for bio-electrosprays to directly handle human sperm without compromising their viability, while also demonstrating the technology's capacity to encapsulate human sperm. These investigations reported herein present interesting implications to human reproductive science and medicine, while also having promising applicability to areas such as the agriculture and aquaculture industries. 


\section{Introduction}

There are many approaches to handling living cells, these range from the direct to the non-direct methods. ${ }^{1}$ Briefly the direct methods are those that mix together all the constituents of tissues, namely the multiple cell types together with components of the extracellular matrix such as collagen to deposit into an architecture of a desired tissue. Conversely non-direct approaches adopt the deposition of a cell friendly/signaling material in a given architecture, subsequently introduced to cells which later are found to engulf that cell friendly architecture, thus forming a cellularised construct. The former is most desirable as it constitutes the least number of steps which would not only significantly reduce contamination issues but would also lower the time taken for creating a fully functional three-dimensional tissue to its implantation. There are many other positive aspects which the direct approaches offer over the non-direct methods. ${ }^{2}$ Hence, in the category of direct approaches, there are many techniques for handling cells, these range from 3D bioprinting techniques to bio-electrosprays, cell electrospinning to others such as aerodynamically assisted biojetting/threading. ${ }^{3}$ In the 3D bioprinting approaches, there are many manifestations to have shown promise, unfortunately those techniques have limitations negating the creation of tissues with the required densities of both cell types and proteins to other tissue constituents as those found in native tissues. This has been limited primarily by the 3D printing technologies, approaches themselves, which have limits on the processable cell densities and types to others, which have shown to cause proteins to denature to inflicting apoptosis and cell death. ${ }^{4}$ Therefore, these technologies do not possess the ability to create a tissue for transplantation in both the laboratory and clinic. On the other hand, bio-electrosprays, cell electrospinning and aerodynamically assisted biojetting/threading, have demonstrated its ability to overcome all the obstacles faced by $3 \mathrm{D}$ bioprinting, but have undergone thorough viability studies from the genetic, genomic to physiological level, which have assessed their possibilities for entering the clinic. ${ }^{5}$ In fact, bio-electrosprays have recently entered the clinic 
for combatting autoimmune diseases such as type 1 diabetes. ${ }^{6}$ In addition, this platform is currently in the process of being assessed for addressing a multitude of regenerative medicine applications in terms of its ability to directly create living fully functional three-dimensional tissues. However, in this communication the authors will focus on bio-electrosprays as applied to the direct handling of human sperm.

Bio-electrosprays (BES) unveiled in 2005 by Jayasinghe et al., ${ }^{7}$ have since been shown to have no negative effects on cells and their behavior in comparison to controls, post exposure over long periods of time. This technology has been applied to a wide range of cell types spanning the immortalized, primary to stem cells including whole fertilized embryos. ${ }^{8}$ In these studies we wish to demonstrate our investigations into bio-electrospray's ability to handle human sperm. Our curiosity is not only triggered by bio-electrospray's flexibility and our accumulated knowledge on the technique, but also by our awareness of the escalating issues currently faced in human reproductive science and medicine. Despite a number of assisted reproductive technologies constantly being introduced or evolving for the improved and safer treatment of infertile patients, those current methods have failed to address the poor success rates of human sperm preservation where a large proportion of sperm is damaged during the freezing and thawing process due to various factors that can affect sperm viability such as temperature changes, ice formation during freezing or thawing and osmotic stress from the cryoprotective solutions that are used in the process. ${ }^{9}$ This is a major disadvantage for men with poor semen parameters as those account for $40-50 \%$ of all infertility cases. ${ }^{10}$ This also highlights the fragility of this specialized cell and the importance of using it in this research model to suggest new alternatives for its preservation. Therefore bio-electrosprays offer a route to both forming encapsulations of multiple compartments, ${ }^{11}$ which could be formed with either separated material membranes, to those of a mixed membrane, within a single encapsulation. This enables the possibility to both precisely control freezing and thawing of sperm samples without 
damage/loss during the process. Therefore, in addressing our curiosity, the first step was to understand whether human sperm could be bio-electrosprayed, without causing any damage, to studying the operational conditions of bio-electrosprays for processing these self-propelling cells in both a medium favorable for maintaining their viability, and for forming nearmonodispersed sperm bearing encapsulations.

\section{Experimental}

Human sperm: Semen samples provided kindly by consented healthy human donors through the GENNET City Fertility clinic in London United Kingdom. Neat and prepared semen explored in these studies were formulated by density gradient.

Computer Assisted Semen Analysis (CASA): Semen analysis was carried out using the IVOS II Hamilton-Thorne Research CASA system (Beverly, MA) with the Motility software HT CASA II version 1.10. In summary, a sperm sample is placed in a fixed depth chamber, examined microscopically and imaged with a digital camera. The HT CASA II software records a series of images $(n=30-45)$ of the field using a rate of 60 images per second. The sperm swim freely in the $\mathrm{x}-\mathrm{y}$ direction, while $\mathrm{z}$-motion along the optic axis is limited by the chamber ceiling and floor. The concentration of sperm and their motility, velocity motion and morphometry parameters are derived for each sperm individually. The sperm tracks are classified according to their velocity and motion parameters and reported to the user. The quality of the specimen is determined from these parameters. 
Semen solution preparation for bio-electrospraying: Human semen solutions were mixed in combinations of alginate and laminin which were mixed in phosphate buffer saline. The alginate explored in these studies were provided by FMC biopolymer, Norway. These explored alginates are ultrapure alginates which are used for biomedical and pharmaceutical applications. The laminin (human recombinant laminin 521) used in these experiments were provided by BioLaminin, Sundbyberg, Sweden. Both these solutions were prepared in their many concentrations (the alginate and laminin concentrations were varied from $1-5 \%$ and $1-10 \%$ respectivly), and added and mixed with either the as neat or prepared samples. The mixed samples were then exposed to bio-electrosprays in a wide range of applied voltage-to-flow rates and imaged using a high-speed camera (Phantom V7, Photo-sonics International Ltd, Oxford, UK).

Bio-electrospraying: Sperm cells were handled using a single needle bio-electrospray setup. In summary the single needle bio-electrospray setup had an internal bore diameter of $\sim 1000 \mu \mathrm{m}$ with a wall thickness of $\sim 700 \mu \mathrm{m}$ with the ground electrode placed at $\sim 5 \mathrm{~cm}$ below the needle. The needle was connected to a DC high voltage power supply manufactured by Glassman Europe Ltd., (FP-30, Tadley, U.K.), while the flow rate was modulated with a syringe pump manufactured by Harvard Apparatus Ltd., (PHD 4400, Edenbridge, U.K.) An applied voltage and flow rate regime of $\sim 1 \mathrm{kV}$ to $\sim 20 \mathrm{kV}$ and $\sim 10^{-9}$ to $\sim 10^{-12} \mathrm{~m}^{3} \mathrm{~s}^{-1}$ was investigated respectively in these studies. The bio-electrospray system was housed in a class II laminar flow hood to maintain sterility. 


\section{Results and discussion}

The first step was to explore bio-electrosprays in the single needle configuration, for assessing whether these cells would survive the exposure to high voltages and collection in comparison to control cells. Note that control cells (CTRL) are those cells that have not been exposed to the bio-electrospray process. Cell suspensions exposed to bio-electrospraying are indicated as BES. A process operational window ranging from $\sim 1 \mathrm{kV}-\sim 20 \mathrm{kV}$ for the applied voltage to the flow rate ranging from $\sim 10^{-9}-\sim 10^{-12} \mathrm{~m}^{3} \mathrm{~s}^{-1}$ was investigated. In addition, we varied the ring ground electrode diameter and its distance away from the exit of the needle, for assessing any effects on either the processed sperm or on the BES process. The human sperm was provided in two different solution configurations namely, neat, and as prepared samples. The neat samples refer to samples that were fresh as collected from patients, while the prepared samples indicate those that are suspended in phosphate buffer saline (PBS). The neat samples on exposure to BES was seen to elongate similar to electrospinning a high viscosity polymer which was later found to attach to either the ring ground electrode or the inlet of the collecting sterile falcon tube. The attachment point varied from either the ground electrode to the top of the flacon tube, as a result of the position of the BES needle with respect to the ground electrode and the falcon tube. These bio-electrospray results did not alter very much for these samples in terms of their jet/fiber behavior under the electric field. The only observation was that for a given equipment set-up if the applied voltage was kept constant and the flow rate was increased, the extending filament was seen to increase in diameter. The converse was observed if the flow rate was constant and the applied voltage was increased. However, the collected semen was analyzed using a computer assisted semen analyzer (CASA) system, showing that post-treated cells were indistinguishable when compared with those control cells. Having assessed the cells, the neat semen samples were mixed with $2 \%$ of PBS, and were subsequently subjected to bioelectrosprays. This mixed semen solution was found to possess the ability to form an unstable 
cone and jet(s), which were later seen to break up into semen-containing droplets. Figure 1 depicts the as collected semen mixed with $\sim 2 \%$ PBS, bio-electrospraying at an applied voltage of $\sim 11 \mathrm{kV}$ for a flow rate of $\sim 10^{-9} \mathrm{~m}^{3} \mathrm{~s}^{-1}$. High speed photography showed the unstable multiple jets forming from a single filament of semen suspension exiting the BES needle (figure 1, arrow mark 1). From previous literature ${ }^{12}$ we see that this process which is unstable promotes the production of a polydisperse distribution of semen-containing droplets, which is also captured in the image (figure 1). In addition, the evolving jets are found to be undergoing jetting modes referred to as stable cone-jetting (figure 1, arrow mark 2) and ramified (figure 1, arrow mark 3) modes. An interesting jetting behavior we have captured during this process was where a completely detached jet from the main filament continues its flight during which the detach jet segment is seen to breakdown into droplets (figure 1, arrow mark 4). This detached jet segment fragmenting into droplets results from the loss in connectivity to the primary filament (figure 1, arrow mark 1) of liquid at the needle exit. 


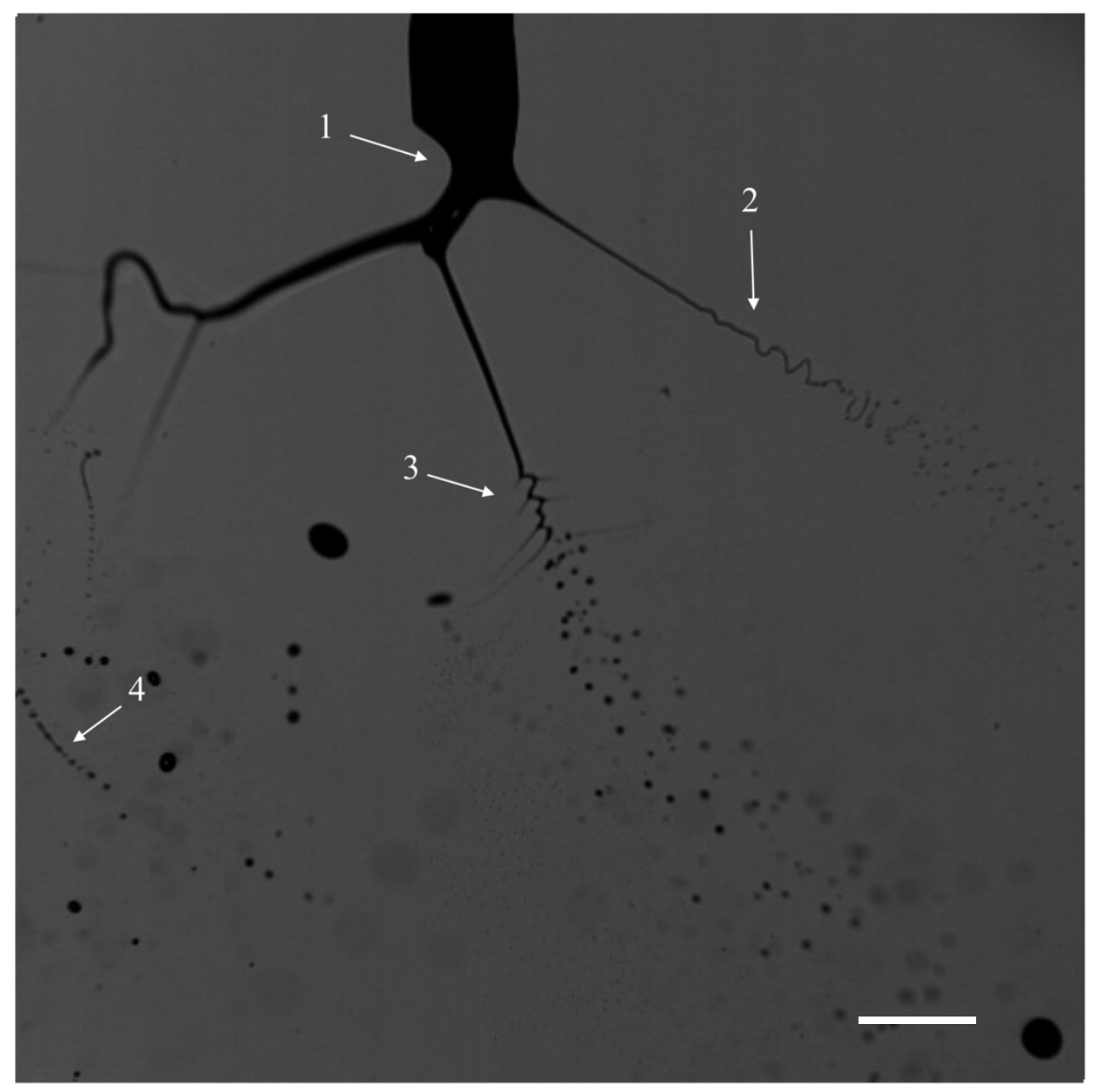

Figure 1. Characteristic high-speed digital image captured using a Phantom V 7.3 high speed camera. Capturing was carried out at $\sim 15000$ fps. Arrow mark 1) depicts the filament of suspension containing sperm, 2) stable cone-jetting with the jet undergoing whipping and transitioning to droplets, 3) ramified jetting with droplets generating from the breaking up of the whiskers formed off the jet to finally 4) a segment of a jet having detached from the primary filament (arrow mark 1), thus loosing electrical connectivity which is later seen to break up into droplets. Scale bar represents 1700 um.

This chaotic jetting behavior was seen to be random if the applied voltage was elevated with the frequency of whipping increasing. If the applied voltage was kept constant and the flow rate was increased, this would cause jetting but would reduce in whipping frequency with the 
increasing flow rate. Nevertheless, stable cone-jetting as a single jetting mode was never achieved. From our past experience in the handling of living cell suspensions this is a direct result of the sample's unbalanced properties of primarily electrical conductivity and viscosity. Therefore, having established that processed cells are seen to remain viable post-treatment, at both varied applied voltage and flow rates for given constant flow rates and voltages respectively, we set out to establish stable cone-jetting in the single jet mode. As one of our intentions was to encapsulate sperm, having now established that the process does not have any negative effects on the sperm, we decided to include alginate in the semen mixture which has previously been explored for compartmentalizing a wide variety of living cells. ${ }^{14}$ Furthermore, we added laminin into the mix so that the matrix properties (such as the rheological properties) within the mixture would be enhanced. Therefore, in the proceeding studies we varied the percentage of alginate mixed in PBS from 1-5\% and also included 2-10\% of laminin.

Medical grade alginate PRONOVO UP MVM supplied by FMC Biopolymer (Norway) and human recombinant laminin 521 (BioLamina, Sundbyberg, Sweden) was mixed with PBS at varied concentrations, and was mixed with prepared samples of human sperm and exposed to bio-electrosprays. At the lowest concentrations of mixtures, the sperm was seen to oscillate from unstable to near stable cone-jetting conditions (figure 2). The equipment arrangement was set to the needle exit having a distance of $\sim 5 \mathrm{~cm}$ away from the ring ground electrode. 
a)

d)

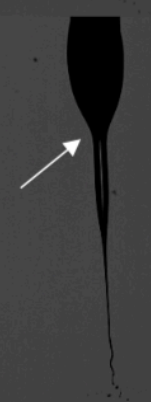

g)

j)

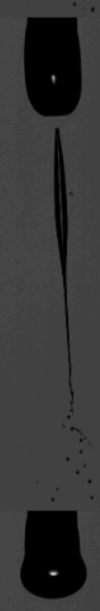

h)

e)

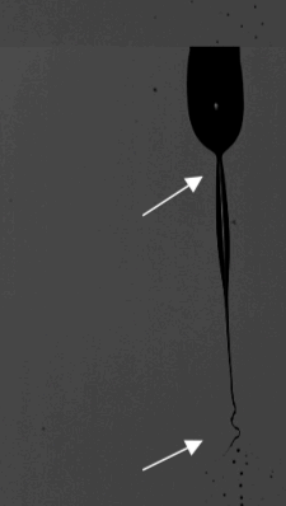

f)

c)

b)

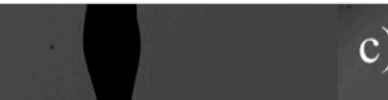

)

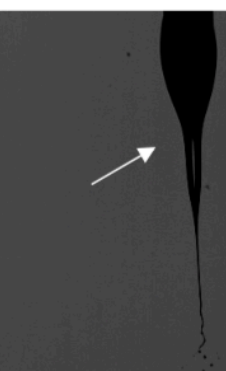

)

f)

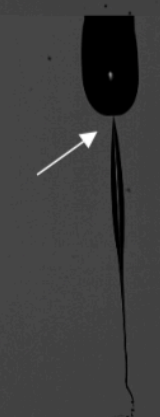

i)

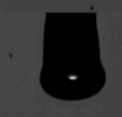

k)

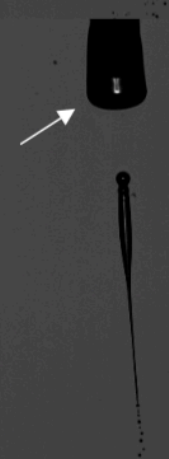

)

1)

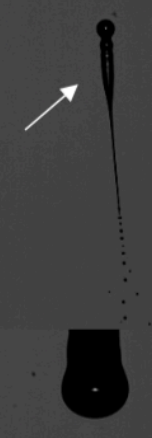

?

Figure 2. Representative captured digital images of the near-stable jetting process, achieved during the bio-electrospraying of human sperm with $1 \%$ alginate mixed with $2 \%$ laminin and human sperm. The panels from a) - 1) depict the achievement of instantaneous single stable 
cone and jet forming at the exit of the needle subsequently resulting in the cone elongating into a large filament of liquid, later detaching from the recoiling cone at the needle exit. The detaching end of the large filament similar to the liquid at the exit of the needle is found and seen to recoil and cause a rippling-like liquid filament while the opposite end is jetting and generating droplets of near mono-dispersity. The loss in electrical connectivity of the detached fragment of liquid while undergoing this rippling or oscillatory behavior is seen to detach from the droplet generating jet and forms into a single large droplet, while the remaining jet on generating droplet soon after detachment are seen to fragmentate into droplets much like in figure 1 arrow mark 4. Scale bar denotes 1700um.

Figure 2 depicts the sequence of digital images captured during the instantaneous forming of the near stable cone-jetting sequence (instability) which was repetitive. The applied voltage and flow rate at this stage was set to $\sim 8 \mathrm{kV}$ and $\sim 10^{-10} \mathrm{~m}^{3} \mathrm{~s}^{-1}$ respectively. The sequence of events captured show the stable cone and jet form, and generate a steady jet from which droplets are generated. This stability evolves into an unstable scenario where the cone section elongates (figure 2, panels b-d) and collects into a larger jet which continues to produce a stable jet and the formation of a near-mono dispersity of droplets. This elongating cone is later seen to narrow and pinch off yet continuing to maintain a stable jet and form droplets (figure 2, panels e- $\mathrm{g}$ ). In panels f) and g) the liquid cone detaches with the elongating cone fragment and starts to withdraw towards the needle exit. Consequently, panels h) to j) show the liquid at the exit of the needle recoil with the cone fragment behaving in a similar fashion but retains the jet and the droplet production process. The disconnection of the liquid from the main cone, on further time lapse shown in panels k) and 1), demonstrate the oscillatory recoiling fragment detaching the jet from its apex and forming into a single large droplet while the detached jet generating droplets are seen to subsequently transition from a jet to a droplet line. These sequences of events take form due to the instability in the jetting process together with the loss in electrical 
connectivity with the charged needle. The behavior of the jetting scenario observed till reaching the voltage of $\sim 8 \mathrm{kV}$ for a given flow rate of $\sim 10^{-10} \mathrm{~m}^{3} \mathrm{~s}^{-1}$ was seen to undergo jetting from microdripping to unstable and oscillatory to unstable coning mode. After reaching the jetting scenario just described in detail and captured in figure 2, further elevating the applied voltage was seen to increase the frequency of the scenario captured in figure 2 , to progressing to the spindle and multi-jet modes which all took place in the unstable mode. If the voltage was kept constant and the flow rate was either lowered or increased the stability would be disturbed in terms of it increase in frequency for reducing the flow rate while, in the case of increasing the flow rate, the jetting process was seen to reduce in frequency and transition into a pulsating jet to microdripping from small to larger droplets with the increasing flow rate. This behavior is typical of a medium having unbalanced properties (electrical conductivity, viscosity, density, relative permittivity and surface tension). Note that unlike previous work carried out with electrosprays ${ }^{13}$, these jets are much more complex, as they contain living biological material which are in motion. In addition, the media explored for maintain these living materials contain components which are not favorable for forming stable spray conditions, such as the need for this media to contain high concentrations of ions, which are required for maintaining the cells complicated metabolisms. The bio-electrospray needle was varied in distance from the ring ground electrode from $\sim 1-10 \mathrm{~cm}$. The closer the needle was to the electrode at elevated applied voltages, for a majority of flow rates, discharging was experienced. For distance nearing $10 \mathrm{~cm}$ the applied voltage required elevation and yet did not stabilise the jetting process. In our pursuit for achieving stable cone-jetting we mixed the highest concentrations of alginate and laminin with the prepared sperm samples, namely $5 \%$ and $10 \%$ of alginate and laminin respectively. These samples were subsequently introduced to bio-electrosprays, a similar operational window was traversed and investigated in detail. 
These high concentrations for both alginate and laminin together with the prepared sperm samples, were seen to encourage greater stability, but this time with the notable observation of the jetting process to yield a much more pronounced cone with a longer jet. The bio-electrospray equipment set-up, namely the distance between the two electrodes were maintained at $\sim 5 \mathrm{~cm}$ with the applied voltage and flow rate set to $\sim 8 \mathrm{kV}$ for a given flow rate of $\sim 10^{-10} \mathrm{~m}^{3} \mathrm{~s}^{-1}$. The increase in the percentage of mixed alginate and laminin with the purified sperm samples increased the suspension viscosity which resulted in a very distinctive formation of a cone and jet at the exit of the needle. The viscosity increase was seen to contribute significantly to the ensuing jet by elongating, thus increasing its whipping motion which is due to the accelerating jet between the electrodes, undergoing resistance forces due to air drag caused by the surrounding atmosphere. Although the viscosity increase gave an increase in the length of the jet and its whipping, the droplets generated were polydispersed as evident from the sequence of images in figure 3. It is noteworthy that although the properties of this sperm suspension were very different to the other, which had a significantly lower percentage of both alginate and laminin, they shared one common behavioral characteristic as seen in the sequence of images depicting the jet detaching from the liquid cone. Noticeably the elongating jet fragment did not contain the same volume of liquid but behaved in a similar fashion. Additionally, the whipping jet did somewhat also behave in a similar manner yet with the differentiation of a chaotic whipping and jetting fragment.

Establishing these bio-electrospray operational conditions were as before where we maintained the distance of $\sim 5 \mathrm{~cm}$ between the electrodes and varied the applied voltage and flow rates. In a very similar jetting behavioral pattern similar to those of figure 2 the applied voltage for a constant flow rate and vice versa were seen to give similar results with the noticeable defined cone which formed at the exit of the needle with the much longer elongating jet. The erratic whipping nature of the jet was seen to increase in frequency with increasing applied voltage 
whist this behavior was seen to settle down to instability at rather lower applied voltages to higher flow rate for a given applied voltage.

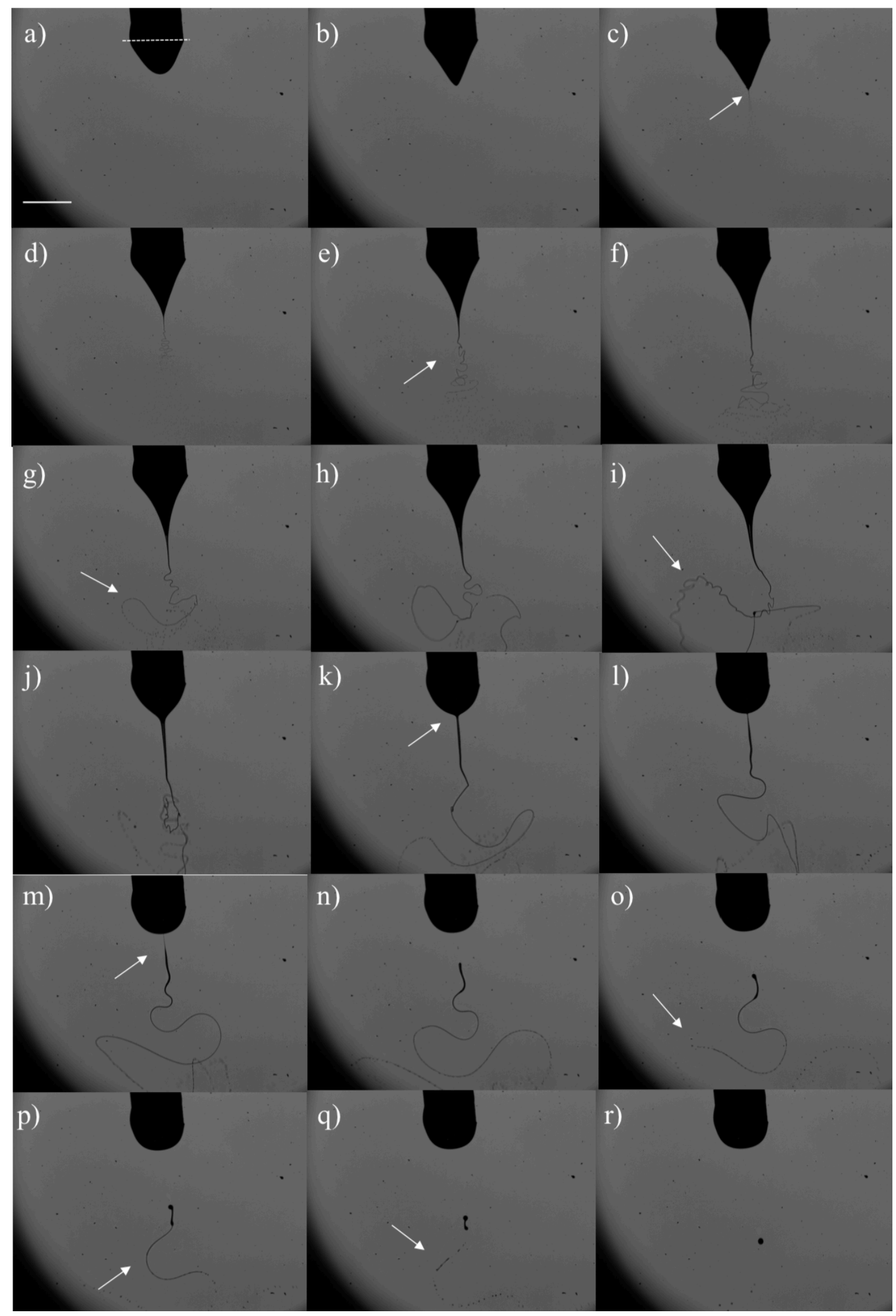


Figure 3. Characteristic digital images of bio-electrospraying in the pulsating cone jet mode, of human sperm with 5\% of alginate mixed with 10\% laminin and prepared human sperm. Panels a) - e) illustrate an evolving pulsating cone and jet forming at the exit of the needle. Note the elongating jet which is fine and later seen to start jet whipping. Sequence in panels f) to i) depict the elongating jet which demonstrates a feature in pulsation. Panels j) - r) show a distinctive dimensional and shape change in the character of the cone whist the jet initially elongate and narrows to subsequently pinching and breaking off. From panels k) to r) the process behavior is very similar to those described and shown in figure 2. Scale bar in panel A) applies to all panels and represents $1700 \mathrm{um}$.

Many samples were studied and closely investigated for their jetting behaviors with varied percentages of both alginate and laminin. In these studies, we subsequently found stable jetting conditions did prevail for the bio-electrospray set up as described above, for an addition of alginate and laminin at $\sim 2.5 \%$ and $\sim 6.5 \%$ respectively, mixed with the prepared sperm samples. Figure 4a portrays the stable cone-jetting process in action at an applied voltage of $\sim 9 \mathrm{kV}$ for a given flow rate of $\sim 10^{-9} \mathrm{~m}^{3} \mathrm{~s}^{-1}$. Increasing the flow rate to $\sim 10^{-7} \mathrm{~m}^{3} \mathrm{~s}^{-1}$ was seen to increase the droplet sizes but was seen to also promote some unstable behavior. At this stage, the applied voltage was elevated to $\sim 10 \mathrm{kV}$. This increase in both flow rate and applied voltage gave rise to a much larger jet diameter, shorter jet and a much more vivid jet whipping behavior (figure $4 \mathrm{~b}$ ). This is not related to the increase in applied voltage as the flow rate was also increased. Moreover, the whipping at the lower voltage, the jet and whipping sections of the jet were similar in length (figure 4a) but in the latter operational condition the whipping jet section was much longer than the jet (figure $4 b$ ). Previous literature does not explain this behavior, however from our experience with these jets we believe this is brought about by the complexity of these matrix rich solutions accommodating living cells, which are studied less than previous studies on suspensions containing non-living materials. 

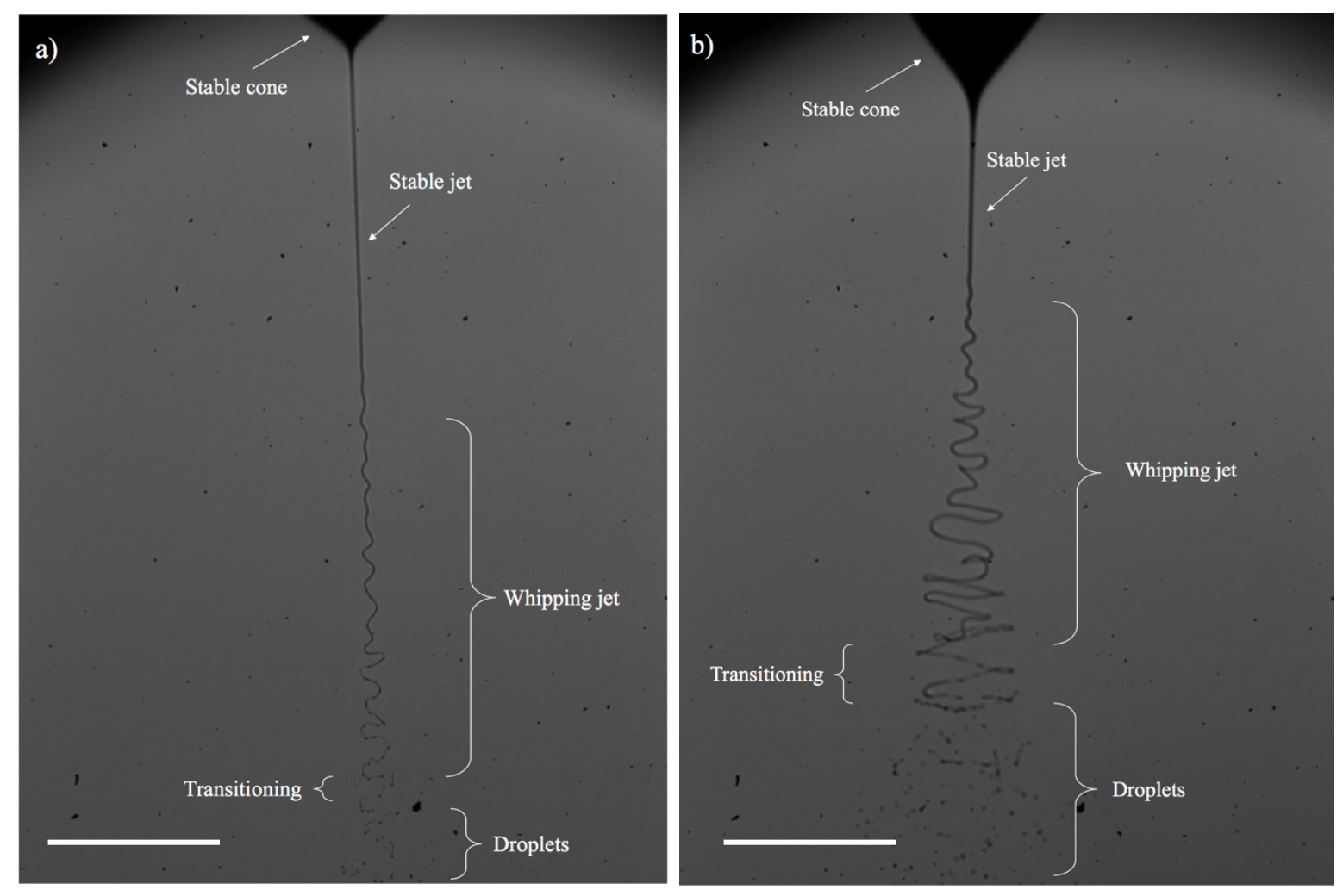

Figure 4. Typical high-speed digital images captured at a) an applied voltage of $\sim 9 \mathrm{kV}$ for a flow rate of $\sim 10^{-9} \mathrm{~m}^{3} \mathrm{~s}^{-1}$ and $\mathrm{b}$ ) at an applied voltage $\sim 10 \mathrm{kV}$ for a given flow rate of $\sim 10^{-7} \mathrm{~m}^{3} \mathrm{~s}^{-1}$. These conditions are seen to have effect on the jet length and diameters, which increases with the increasing flow rate. A notable feature previously not reported clearly in the literature, is the proportions in the lengths in the jet compared to that of the whipping section of the jet. Commonly the whipping section is shorter than the jet and in some scenarios, despite being longer, is seen to frequently detach after formation from the jet and breaks down into droplets. This was not the case here. Therefore, this feature is attributes to the properties of these living suspensions for maintaining the well-being of the human sperm. Scale bars in panels a) and b) represent 1700um.

Since achieving stable cone-jetting mode, all subsequent samples of prepared sperm were mixed at these proportions of alginate and laminin and bio-electrosprayed. The resulting droplet were collected directly into sterile falcon flasks. The collected sperm samples were immediately 
analyzed using the CASA system for assessing the various aspects of sperm viability in comparison to controls. Figure 5a shows the collected sperm samples in comparison to control samples (figure 5b). 

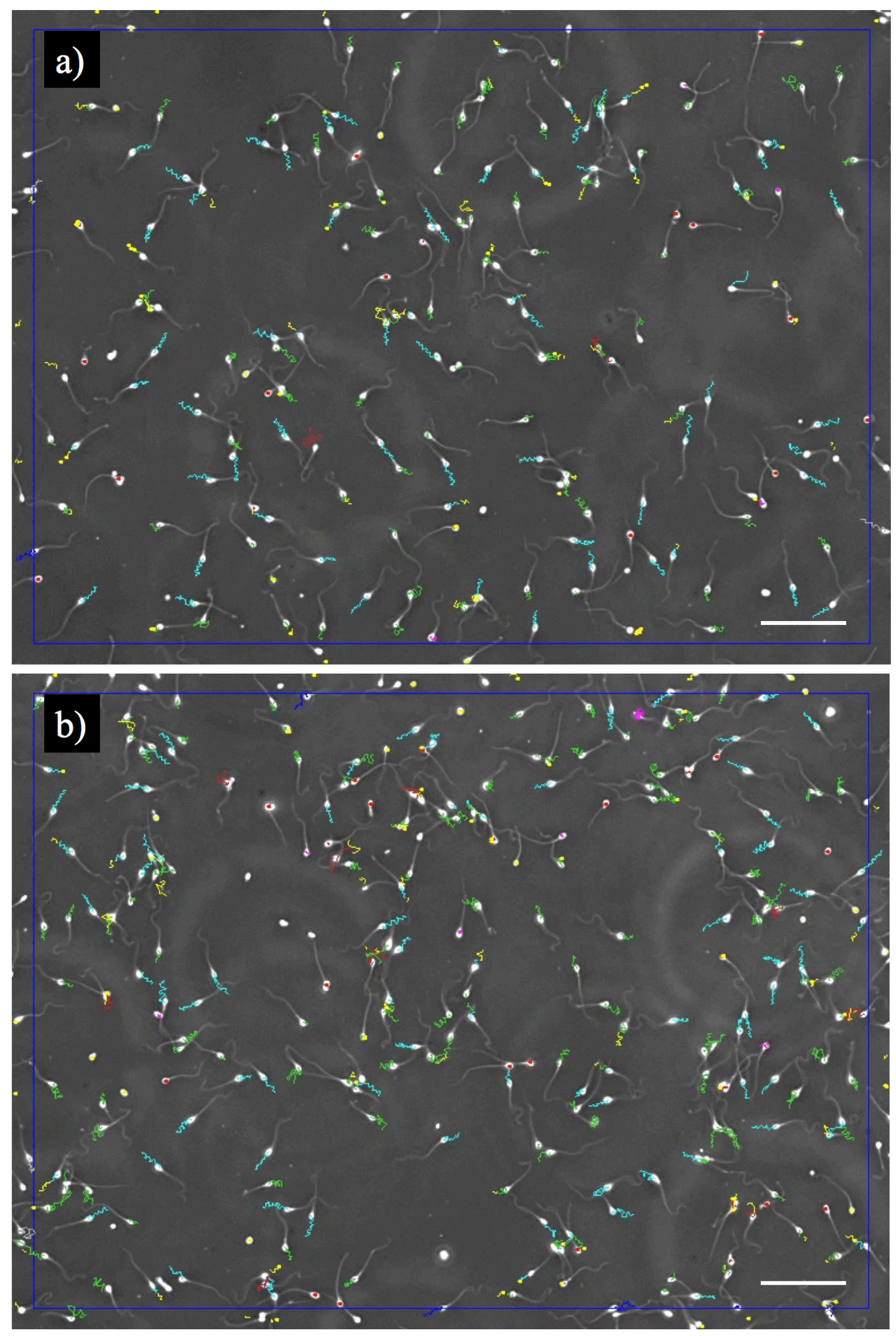
Figure 5. Representative optical micrographs of a) bio-electrosprayed (BES) and b) the control (CTRL) sperm cells. On observing the samples, they were indistinguishable from each other. Both samples were seen to have a significantly large proportion of live sperm cells. The colour coded lines seen on the heads of the spermatozoa are the trajectories followed and generated on the CASA system for assessing their different motility classifications according to the World Health Organisation (WHO) guidlines. Note that immotile spermatozoa are identified with a red dot and very few cells contain these dots instead of trajectory lines in both panels. Scale bars in both panels represent 50um.

On assessment of samples through the CASA system, we noted that a large majority were progressively motile in all the collected (56\% category A and $20 \%$ category B motility sperm, Figure 6a) and control samples (52\% category A and $20 \%$ category B motility sperm, Figure 6a), no statistical significance was noted between the two groups. Further analysis of all velocity motion parameters of spermatozoa again highlighted no significant differences between collected and control samples (Figure 6b); average velocity path (VAP, 33.7 vs $32.0 \%$ respectively, NS), curvilinear velocity (VCL, 62.6 vs $59.9 \%$ respectively, NS), straight line velocity (VSL, 23.1 vs $21.6 \%$ respectively, NS), and amplitude of lateral head displacement (ALH, $4.6 \%$ for both groups, NS), and no significant differences in terms of proportion of spermatozoa that are hyperactivated (5.1 and $4.6 \%$ respectively, NS, Figure $6 \mathrm{c})$. 
a)

WHO Motility Distribution

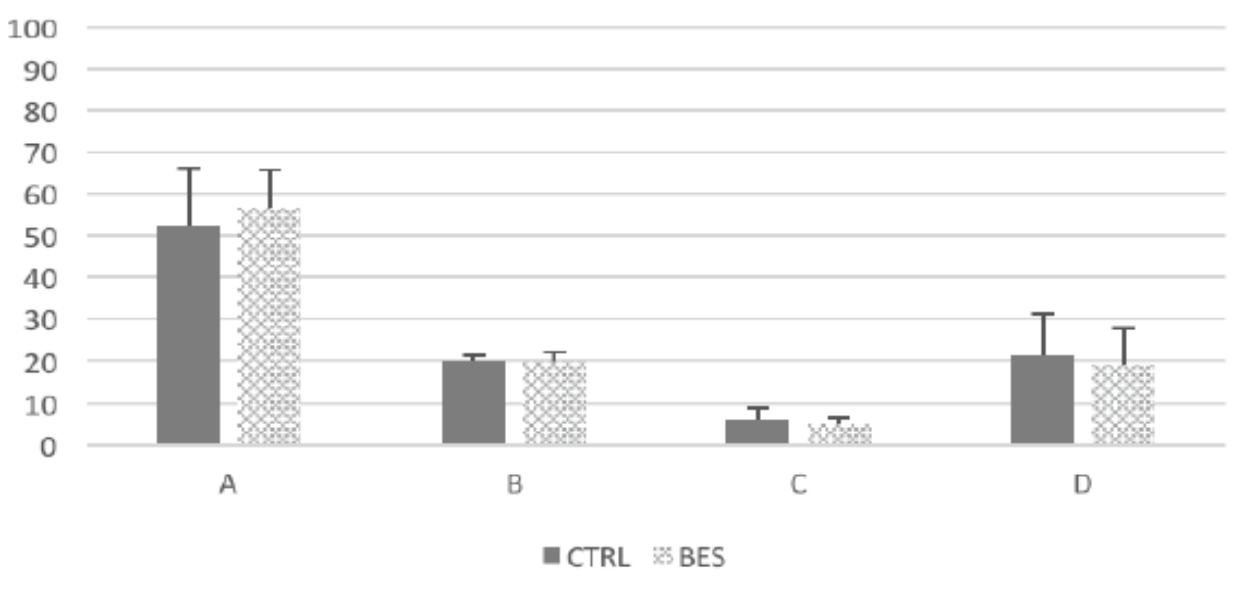

b)

Mean Sperm Kinetics

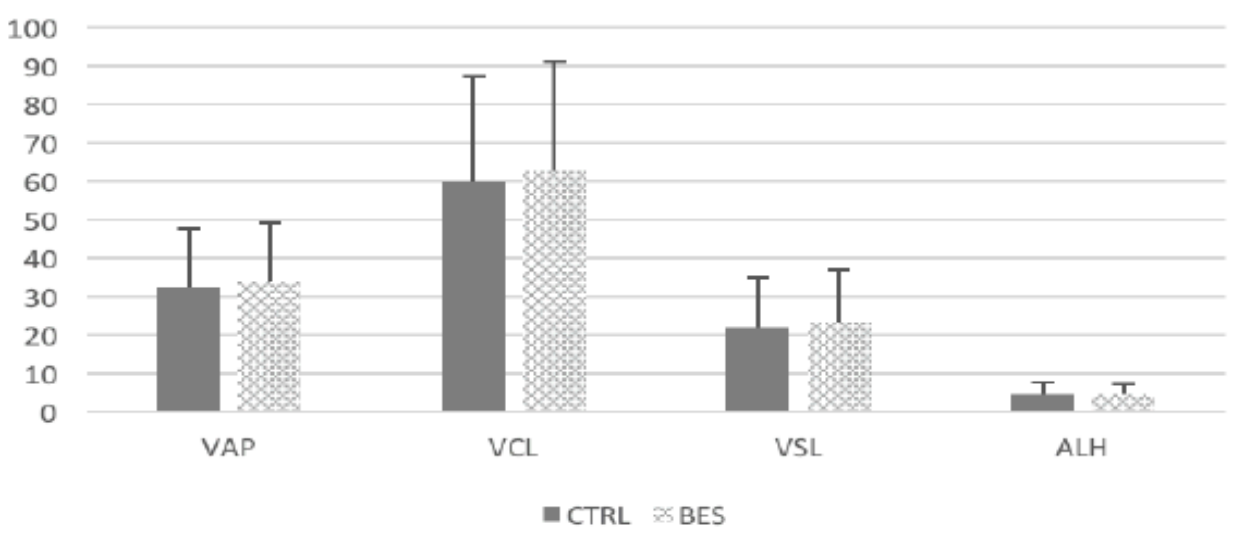

c)

\% Hyperactivation

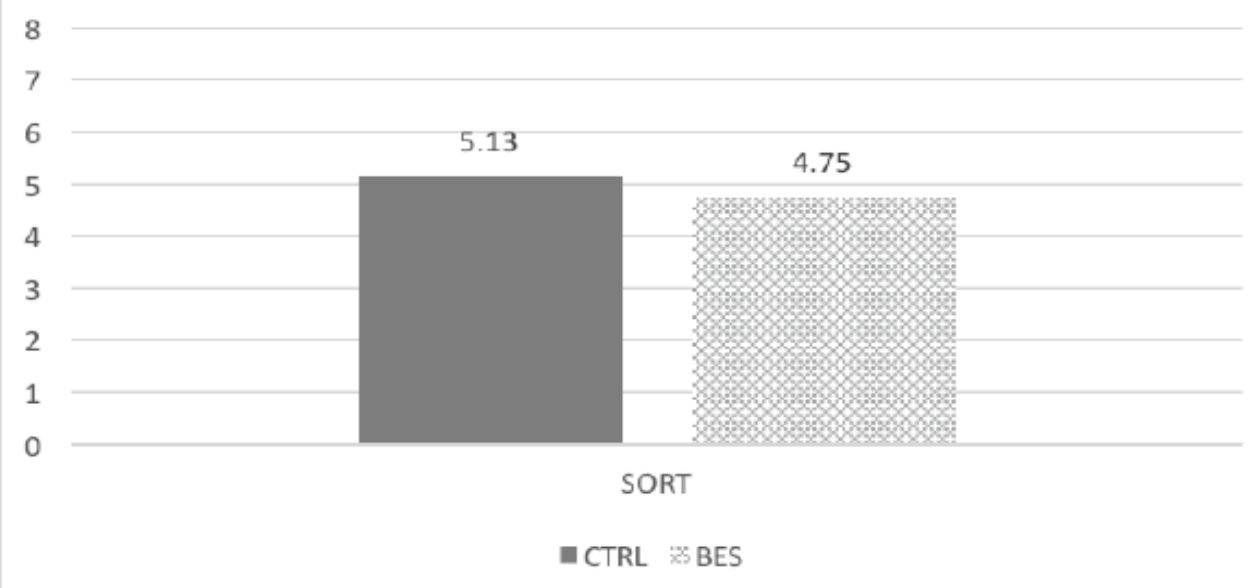


Figure 6. Summary graphs representing a) motility according to WHO classifications of spermatozoa between BES and control samples, b) classification of sperm velocity motion parameters between BES and control samples, and c) hyperactivation index sub-population of spermatozoa in BES and control samples, no significant differences observed in any of the groups, NS $>0.05$.

The post-bio-electrosprayed cells were comparable in all the assessed aspects in term of their viability. Therefore, our investigations progressed to encapsulating the sperm which was carried out with calcium chloride. In this instance, the bio-electrospray equipment set up was modified, removing the sterile falcon flask (previously used for sample collection) and was replaced with a magnetically stirred beaker accommodating the calcium chloride. The concentrations of calcium chloride explored in these studies were similar to those explored in our previous studies. ${ }^{14}$ Cross-linked and collected samples were observed and were seen to be indistinguishable with control samples. Figure 7a shows the encapsulated spermatozoa within a cross linked bead of alginate. Closer examination of the bead showed the encapsulated sperm within the bead (figure $7 b)$. 

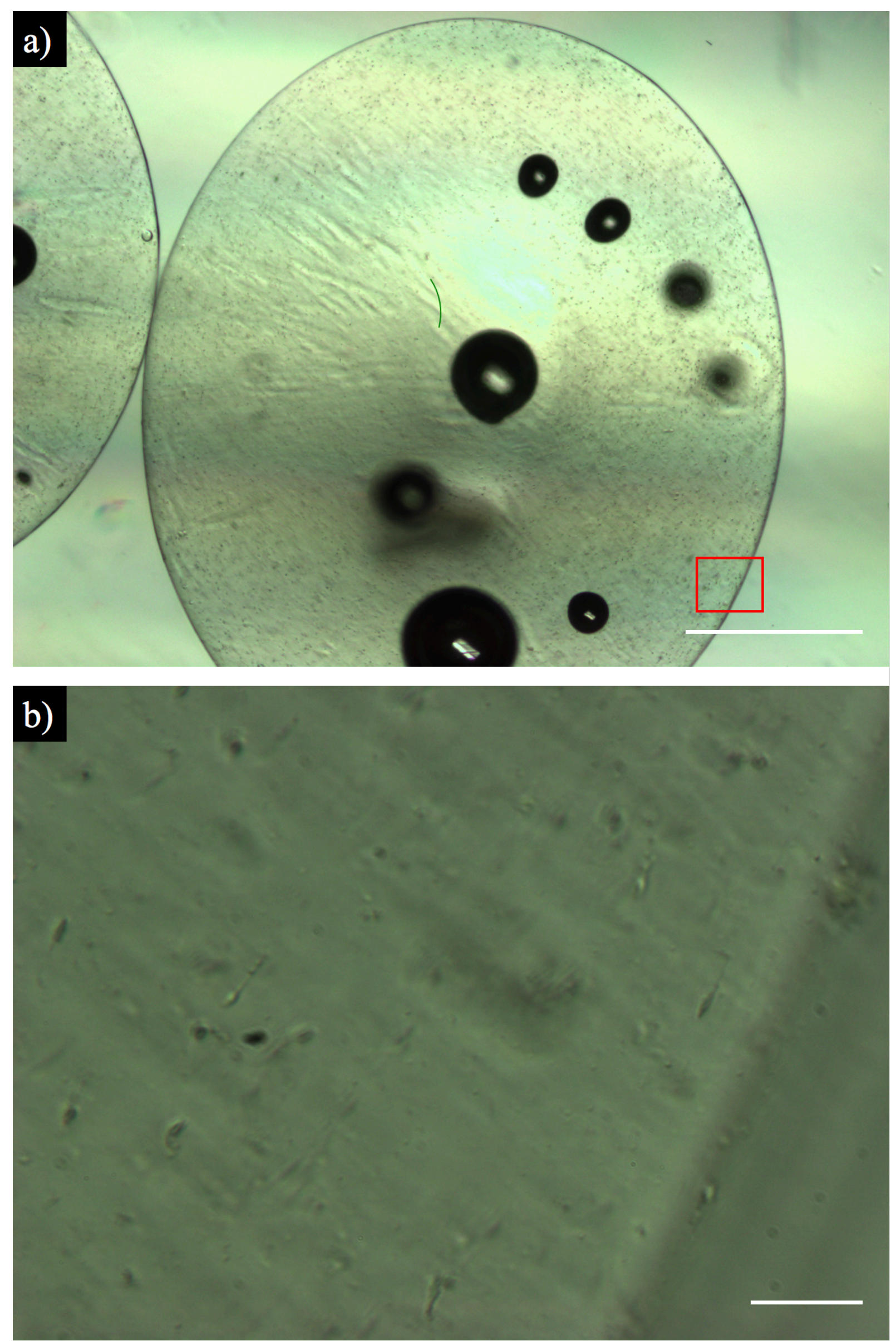
Figure 7. Optical micrographs depicting characteristics images of a) semen encapsulating microbead and b) a high magnification image of the compartmentalized semen. Scale bars in panels a) and b) represent $1 \mathrm{~mm}$ and 50um respectively.

In figure 7a the fine specks which appear to be dispersed within the bead are the spermatozoa, the relatively large darker spheres are trapped bubbles within the bead. Note that some of these specks which are the spermatozoa, and these bubbles appear to be in focus while some are out of focus, this is due to the focus plane of the microscope, and these encapsulate proximities being in three-dimensions. The magnification in panel $7 \mathrm{~b}$ depicts the dispersed and compartmentalized spermatozoa within the bead, here as in the previous image in panel 7a some of the encapsulates appear to be in focus while other are not. These encapsulates where dissolved and found to have retained the sperm cells viability, as the live cell numbers were comparable to control cells. Figure 8 depicts collected sperm cell encapsulates over a time frame of $\sim 10$ minutes. 


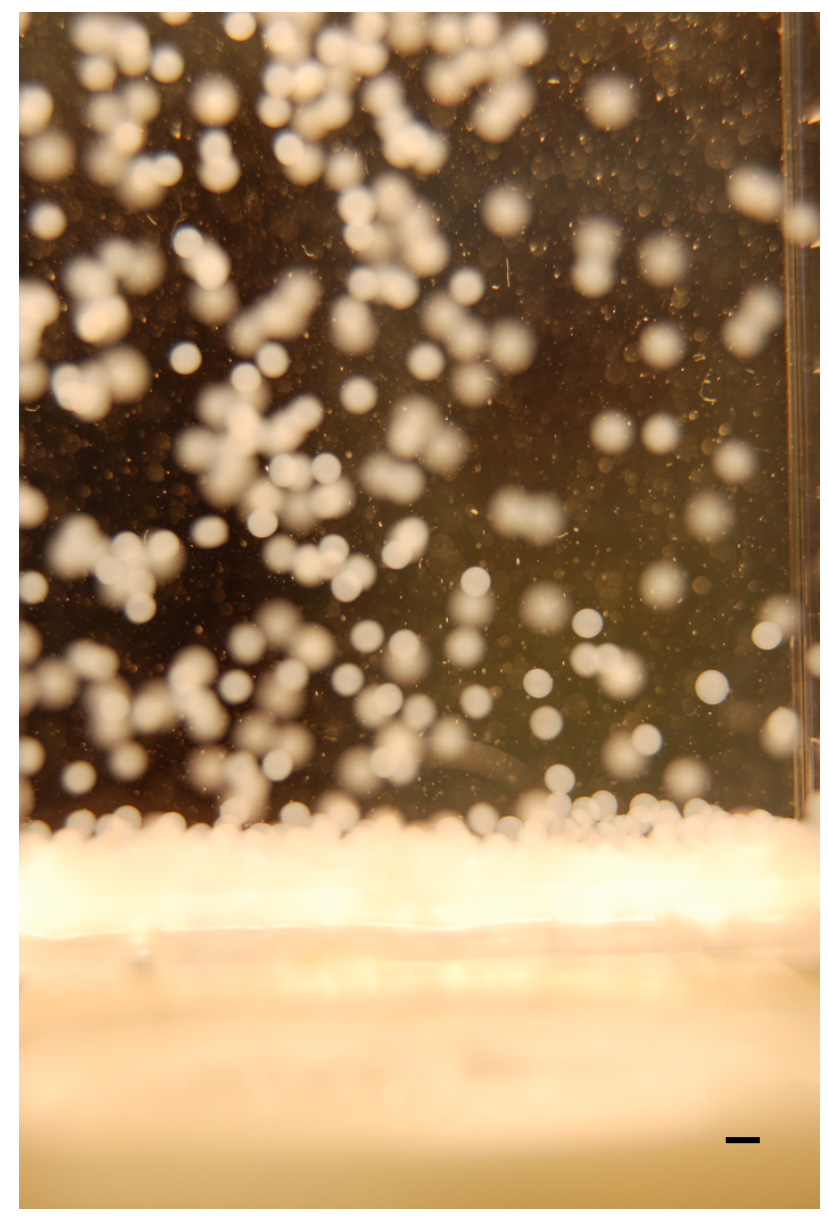

Figure 8. Bio-electrosprayed and compartimentalised semen collected over a time frame of $\sim 10$ minutes. The image depicts those encapsulates resuspensed in phsophate buffer saline. Scale bar represents $\sim 3 \mathrm{~mm}$.

\section{Conclusions}

These studies demonstrate the versatility of bio-electrosprays ability to handle a wide range of living cells. Similar results were observed for cell electrospun human sperm. In these studies, we demonstrated the ability for bio-electrosprays to directly handle human sperm without compromising its viability. Many aspects of sperm viability were assessed using the wellestablished computer assisted semen analysis system. The post-bio-electrosprayed cells were compared with controls, and found to be indistinguishable. In addition to this finding we 
pursued the ability to BES these sperm cells in stable conditions, for generating nearmonodispersed sperm containing crosslinked beads, which could be applied to many purposes in the reproductive science and medicine fields of research and development. For example, where patients with low sperm counts, could have semen encapsulated and stored for use at a later date, at which time the harvesting of these cells would be susceptible to minimum or no loss in cell viability. This would be controlled by the capacity for cells to be compartmentalized in multiple compartments which would allow the encapsulated spermatozoa to be frozen and thawed without inflicting any negative aspects on those encapsulates. This application is also relevant in fields such as the agriculture and aquaculture industries where sperm could be sorted and used when required without any losses. Thus, enabling the maintenance of a longer shelflife for encapsulated spermatozoa and seeds. 


\section{Conflicts of interest}

There are no conflicts to declare.

\section{Acknowledgements}

SNJ thankfully acknowledges funding provided by the Royal Society through their seed corn funding mechanism. The authors thank Dr. Shaun Rogers at Gennet City Fertility clinic for supplying the human sperm explored in these studies. 


\section{References}

1 a) S.N. Jayasinghe, Regenerative Medicine. 2008, 3, 49; b) C. Ru, J. Luo, S. Xie and Y. Sun, J. Micromech. Microeng. 2014, 24, 053001.

2 a) S.N. Jayasinghe, Biomedical Materials, 2008, 3, 034004; b) H. Cui, M. Nowicki, J.P. Fisher and L.G. Zhang, Adv. Healthc. Mater., 2017, 6, 1601118.

3 a) S. Vijayavenkataraman, W-C. Yan, W. F. Lu, C-H. Wang, J. Y. H. Fuh, Advanced Drug Delivery Reviews, 2018, https://doi.org/10.1016/j.addr.2018.07.004.b) P.A.M. Eagles, A.N. Qureshi and S.N. Jayasinghe, Biochemical Journal, 2006, 394, 375; c) S.N. Jayasinghe, S. Irvine and J.R. McEwan, Nanomedicine, 2007, 2, 555; d) S. Arumuganathar, S. Irvine, J.R. McEwan and S.N. Jayasinghe, Biomedical Materials, 2007, 2, 158; e) S. Arumuganathar, and S.N. Jayasinghe, Biomedical Materials, 2007, 2, 189.

4 a) G. M. Nishioka, A. A. Markey, and C. K. Holloway, J. Am. Chem. Soc., 2004, 126, 16320; b) K. Nair, M. Gandhi, S. Khalil, K. C. Yan, M, Marcolongo, K. Barbee and W. Sun, Biotechnology Journal, 2009, 4, 1168; c) L. Ning, N. Betancourt, D. J. Schreyer, and X. Chen, ACS Biomaterials Science \& Engineering, 2018, 11, 3906-3918.

5 a) A. Eddaoudi, A. Townsend-Nicholson, J. Timms, S. Schorge and S.N. Jayasinghe, Analyst, 2010, 135, 2600; b) R. P. Hall, C. M. Ogilvie, E. Aarons and S.N. Jayasinghe, Analyst, 2008, 133, 1347; c) E. Ehler and S.N. Jayasinghe, Analyst, 2014, 139, 4449.

6 B. E. Tuch, G. W. Keogh, L. J. Williams, W. Wu, J. L. Foster, V. Vaithilingam, R. Philips Diabetes Care, 2009, 32, 1887.

7 S.N. Jayasinghe, A.N. Qureshi and P.A.M. Eagles, Small, 2006, 2, 216.

8 a) J.D.W. Clark and S.N. Jayasinghe, Biomedical Materials, 2008, 3, 011001; b) T. Geach, N. Mongkoldhumrongkul, L.B. Zimmerman, and S.N. Jayasinghe, Analyst, 2009, 134, 743; c) P. Joly, B. Hennings, and S.N. Jayasinghe, Biomicrofluidics, 2009, 3, 044107; d) N.K. Pakes, S.N. Jayasinghe and R.S.B. Williams, Journal of the Royal Society, Interface, 2011, 8, 1185. 
9 M. Bell, R. Wang, W. J. Hellstrom, and S. C. Sikka Journal of Andrology, 1993, 14, 472.

10 Yen and Jaffe's Reproductive Endocrinology (Eighth Edition), Editor(s): Jerome F. Strauss, Robert L. Barbieri, 2019,

11 Z. Liu, H.C. Shum. Biomicrofluidics. 2013, 7, 44117.

12 A. Jaworek and A. Krupa, J. Aerosol Sci., 1999, 30, 873.

13 R.P.A. Hartman, D.J. Brunner, D.M.A. Camelot, J.C.M. Marijnissen and B. Scarlett, J. Aeorsol Sci., 2000, 31, 65.

14 V.L. Workman, L.B. Tezera, P.T. Elkington and S.N. Jayasinghe, Advanced Functional Materials, 2014, 24, 2648. 